\title{
CONCENTRADO DE MELANCIA (Citrullus vulgaris SCHRAD): ACEITAÇÃO SENSORIAL, PARÂMETROS MICROBIOLÓGICOS, FÍSICO-QUÍMICOS E DETERMINAÇÃO DE FITONUTRIENTES
}

\author{
NAYARA MOREIRA LACERDA MASSA * \\ ILKA MARIA LIMA DE ARAÚJO ** \\ MARIA LÚCIA DA CONCEIÇÃO *** \\ CAIO VICTOR COUTINHO OLIVEIRA * \\ JAILANE DE SOUZA AQUINO ***+* \\ MARIA DA CONCEIÇÃO RODRIGUES GONÇALVES ****
}

\begin{abstract}
Objetivou-se elaborar concentrado de melancia e determinar a aceitação sensorial, os parâmetros microbiológicos, físico-químicos e os fitonutrientes do produto. A polpa e a entrecasca foram trituradas e homogeneizadas para aplicação de tratamento térmico até a obtenção do concentrado. Adicionou-se gelatina sem sabor de coloração vermelha em uma parte do concentrado. As amostras foram resfriadas e acondicionadas em temperatura de refrigeração. Foram realizadas análises microbiológicas e sensoriais do concentrado de melancia e do concentrado acrescido de gelatina. A partir dessas análises, selecionouse o concentrado puro para determinação dos parâmetros físicoquímicos e do screening fitoquímico, comparando-o à fruta in natura. Ambos os concentrados apresentaram qualidade microbiológica em conformidade com a legislação brasileira vigente. O concentrado sem adição de gelatina foi selecionado para as demais análises por apresentar boa aceitação e as maiores notas nos atributos cor, sabor, sabor residual e textura. Quando comparado à fruta in natura, o concentrado apresentou menor percentual de umidade, proteína, pectina e acidez. Entretanto, o tratamento térmico concentrou os carboidratos e as cinzas (23,99\% e 1,03\%, respectivamente) aumentando seu valor calórico de 28,05 para 104,86 Kcal. Comprovou-se a viabilidade de produção do concentrado de melancia, cujo princípio ativo está baseado em esteroides e flavonoides. Deve-se considerar a ampla disponibilidade da fruta, suas características sensoriais, nutricionais e a presença de compostos fitoquímicos com propriedades antioxidantes. Esse produto, além de apresentar maior vida-de-prateleira em relação à fruta in natura, representa opção de alimento funcional necessário à dieta saudável.
\end{abstract}

PALAVRAS-CHAVE: MELANCIA; ANÁLISE SENSORIAL; CONCENTRADO DE MELANCIA; FITOQUÍMICOS; Citrullus vulgaris SCHRAD.

* Mestrandos em Ciências da Nutrição, Departamento de Nutrição, Universidade Federal da Paraíba (UFPB), João Pessoa, Paraíba (e-mail: nayaramassa@hotmail.com; caiovco@gmail.com).

** Mestre em Ciências da Nutrição, Nutricionista, UFPB, João Pessoa, Paraíba (e-mail:coape@prap.ufpb. br).

*** Doutora em Ciências da Saúde, Departamento de Nutrição, UFPB, João Pessoa, Paraíba (e-mail: mluciacufpb@yahoo.com.br).

**** Doutora em Ciências da Nutrição, Departamento de Nutrição, UFPB, João Pessoa, Paraíba (e-mail: lalaaquino@hotmail.com).

***** Doutora em Produtos Naturais e Sintéticos Bioativos, Departamento de Nutrição, UFPB, João Pessoa, Paraíba (e-mail: raulceica@ig.com.br). 


\section{INTRODUÇÃO}

A melancia, originária das regiões secas da África tropical, é a Cucurbitácea mais produzida no mundo. No Brasil, as regiões mais indicadas para o plantio da melancia situam-se no Semiárido Nordestino, nas quais podem ser plantadas em qualquer época do ano (CARVALHO, 1999). A fruta representa expressiva importância no agronegócio brasileiro, ocupando o quarto lugar dentre as olerícolas, com produção de 2.052 .928 de toneladas no ano de 2010 (IBGE, 2010).

A melancia constitui fonte de minerais (potássio, magnésio, cálcio e ferro) e aminoácidos (citrulina e arginina), além de ser rica em compostos com propriedades antioxidantes como o licopeno, vitamina C, flavonoides e outros compostos fenólicos (OMS-OLIU et al., 2012; RAWSON et al., 2011). Seu consumo tem sido associado à prevenção de doenças degenerativas, como câncer de próstata, estômago e pulmão (EUCARPIA, 2008), ao tratamento de diabetes mellitus (AHN et al., 2011), da síndrome metabólica (WU et al., 2007) e à redução da pressão arterial (FIGUEROA et al., 2012).

Segundo Dias et al. (2006), a melancia apresenta agradáveis características sensoriais de aroma, cor, sabor e refrescância. A polpa e a casca são utilizadas para a produção de farinhas, produtos desidratados, doces, geleias, bolos, biscoitos e sucos (SANT'ANA e OLIVEIRA, 2005; QUEK, CHOK e SWEDWND, 2007; GUIMARÃES, FREITAS e SILVA, 2010; RAWSON et al., 2011; GUIMARÃES et al., 2012) de forma artesanal, ou em baixa escala industrial. A melancia encontrase entre as frutas que apresentam altos teores de água e que produzem grande quantidade de resíduos como, cascas, entrecascas e sementes (SANT'ANA e OLIVEIRA, 2005). Deve-se ressaltar a necessidade de seu processamento como forma de aproveitamento de resíduos e diminuição do desperdício devido sua alta perecibilidade.

Dada à insipiência de informações na literatura científica sobre produtos elaborados com a melancia e considerando a presença de nutrientes com ações funcionais benéficas à saúde da população, o presente estudo objetivou elaborar concentrado de melancia a partir da fruta in natura e determinar os parâmetros microbiológicos, sensoriais, físico-químicos e screening fitoquímico do produto.

\section{MATERIAL E MÉTODOS}

\subsection{MATÉRIA-PRIMA}

Melancias da variedade Citrullus vulgaris Schrad (10 kg), adquiridas da Empresa Paraibana de Abastecimento e Serviços Agrícolas (EMPASA/PB) e no Mercado Central da cidade de João Pessoa (PB), foram transportadas para o Laboratório de Técnica Dietética do Departamento de Nutrição da Universidade Federal da Paraíba (UFPB).

\subsection{ELABORAÇÃO DO CONCENTRADO DE MELANCIA (Citrullus vulgaris SCHRAD)}

A fruta passou pela etapa de higienização e sanitização em solução de hipoclorito de sódio 200 ppm de cloro residual livre por 15 minutos, com posterior subdivisão e retirada da casca. Após a trituração da polpa e da entrecasca efetuou-se a homogeneização e cocção desses componentes à temperatura de $80^{\circ} \mathrm{C} \pm 7$, durante 50 minutos, até a obtenção do concentrado. Adicionou-se gelatina sem sabor com coloração vermelha a uma parte do concentrado à base de melancia obtido. Ambos os concentrados foram deixados em temperatura ambiente e posteriormente acondicionados em embalagens descartáveis, devidamente vedadas e armazenadas à temperatura de refrigeração $4{ }^{\circ} \mathrm{C}$. Calculou-se o rendimento do concentrado pela relação percentual entre o peso da fruta inteira e o peso do concentrado obtido (ARAÚJO, 1999). 


\subsection{ANÁLISES MICROBIOLÓGICAS}

Para as determinações microbiológicas do concentrado de melancia (Citrullus vulgaris Schrad) foram homogeneizados $10 \mathrm{~g}$ de cada concentrado em $90 \mathrm{~mL}$ do diluente de água peptona tamponada a $0,1 \%$, sendo obtidas as diluições decimais de $10^{-1}, 10^{-2}$ e $10^{-3}$ a partir da primeira. As amostras foram analisadas em triplicatas quanto à contagem total de bactérias aeróbias mesófilas, coliforme totais, coliformes fecais termotolerantes, contagem de Staphylococcus coagulase positiva e Salmonella spp. (VANDEZANT e SPLITTSTOESSER, 1992).

\subsection{ANÁLISE SENSORIAL}

Participaram da análise sensorial 59 julgadores não treinados dentre estudantes e funcionários da UFPB, sendo $11,9 \%$ do sexo masculino e $88,1 \%$ do sexo feminino, com faixa etária entre 20 a 40 anos. Todos os julgadores, com nível superior concluído ou em curso, foram recrutados com base no consumo de melancia e disponibilidade em participar do teste. A análise sensorial foi realizada após aprovação da pesquisa pelo Comitê de Ética em Pesquisa com Seres Humanos (CEP/HULW/UFPB), sob o protocolo número 472/11.

Os testes sensoriais foram realizados em cabines individuais do Laboratório de Técnica Dietética, com iluminação artificial uniformemente distribuída, longe de ruídos e odores, em horários previamente estabelecidos, excluindo-se uma hora antes e duas horas após o almoço. Foram utilizadas duas amostras, uma contendo apenas o concentrado de melancia e a segunda o concentrado de melancia acrescido de gelatina (sem sabor e coloração vermelha). Amostras com $5 \mathrm{~g}$ foram servidas em uma sessão, usando-se pratos descartáveis codificados com três digitos aleatórios (MEILGAARD, CIVILLE e CARR, 1988), acompanhadas de biscoito tipo cream cracker, copo de água (para remoção de sabor residual) e das fichas de avaliação.

As amostras foram avaliadas quanto aos atributos de aparência geral, cor, aroma, textura, consistência, sabor, sabor residual e qualidade global, mediante escala hedônica estruturada com nove pontos ( 1 = desgostei muitíssimo e 9 = gostei muitíssimo). Avaliou-se a intenção de compra utilizando escala estruturada com cinco pontos, ancorada pelas avaliações hedônicas: 1 = decididadamente compraria e 5 = decididamente não compraria (MEILGAARD, CIVILLE e CARR, 1988).

\subsection{ANÁLISES FÍSICO-QUÍMICAS}

Todas as análises físico-químicas foram realizadas, em triplicata, no produto mais aceito. As determinações de umidade, resíduo mineral fixo, proteínas, extrato etéreo, pectina, pH e acidez titulável foram efetuadas conforme recomendação do Instituto Adolfo Lutz (BRASIL, 2005). Calculouse o teor de carboidratos pelo método da diferença e o valor energético total conforme o Ministério da Saúde (BRASIL, 2003). As determinações de açúcares redutores em glicose, frutose e não redutores em sacarose foram realizadas de acordo com Ranganna (1979).

\subsection{SCREENING FITOQUÍMICO}

Realizou-se o screening no concentrado com maior aceitação sensorial, bem como em amostras de sementes, polpa e entrecasca da melancia. Efetuou-se a análise qualitativa dos metabólitos secundários (saponinas, esteroides, flavonoides e taninos) presentes na polpa, entrecasca e concentrado da fruta, segundo a metodologia proposta por Barbosa Filho (1997). A extração do material vegetal para a triagem fitoquímica, em base seca e pulverizada, foi realizada com $100 \mathrm{~g}$ de polpa, de entrecasca e de sementes da melancia (Citrullus vulgaris Schrad). A extração ocorreu sob refluxo com álcool etílico comercial filtrado e evaporado em rotavapor para obtenção do resíduo utilizado nas seguintes análises qualitativas: teste de espuma e teste de hemólise para a detecção da presença de saponinas; reação de Liebermann-burchard para a determinação de 
esteroides; reação de Shinoda para detecção dos flavonoides; reação de cloreto férrico a $2 \%$ e reação de gelatina a $0,5 \%$ para a determinação dos taninos.

Os resultados foram qualificados por sistema de cruzes em que: $(++)=$ reação positiva notável, $(+)$ = reação fracamente positiva, $(-)=$ reação negativa ou resultado inconclusivo para cada classe de metabólito secundário avaliada. A interpretação das colorações e/ou precipitado ocorreu por dois avaliadores.

\subsection{ANÁLISE ESTATÍSTICA}

Os resultados das análises físico-químicas e sensoriais foram submetidos ao teste $t$ de Student ao nível de $5 \%$ de significância $(p<0,05)$, utilizando-se o pacote estatístico Sigma Stat versão 3.5 (JANDEL SCIENTIFIC, 2007). Realizou-se a correlação de Pearson para analisar os resultados relativos aos açúcares redutores e não redutores determinados no concentrado de melancia.

\section{RESULTADOS E DISCUSSÃO}

\subsection{RENDIMENTO DO CONCENTRADO DE MELANCIA}

O rendimento do concentrado de melancia, de $28 \pm 2 \%$, foi considerado baixo quando comparado ao de outras frutas, devido ao elevado teor de água presente na fruta in natura e perdido durante o processamento (LUENGO et al., 2000). Apesar de não ser rendimento expressivo, o processo torna-se viável para aumentar a vida-de-prateleira da fruta e aproveitar o resíduo da entrecasca da melancia. Em estudo realizado por Pinto (2002), com frutas minimamente processadas, a melancia evidenciou rendimento entre 29 e $40 \%$, próximo ao determinado para o concentrado elaborado no presente estudo. Já o melão revelou rendimento superior ao da melancia e seus produtos, entre 38 e $42 \%$. Deve-se salientar que apesar do tratamento térmico, o concentrado de melancia apresentou maior rendimento quando comparado ao obtido por Guimarães, Freitas e Silva (2010) na produção de farinhas a partir da entrecasca da melancia $(1,28 \%)$ e de $0,85 \%$ de rendimento constatado por Pereira, Miguel e Carvalho (2010).

Durante o período do armazenamento do concentrado observou-se aumento linear na quantidade de líquido liberado no interior do recipiente, estando em consonância com os estudos de Xisto et al. (2012) avaliando melancia minimamente processada e de Marrero e Kader (2006) analisando fatias de abacaxi.

\subsection{ANÁLISE MICROBIOLÓGICA}

Os resultados das análises microbiológicas para as amostras de concentrado de melancia adicionado ou não de gelatina apresentaram valores menores que $10^{3} \mathrm{UFC} / \mathrm{mL}$ para contagem total de bactérias aeróbia mesófilas, estando de acordo com as exigências do Ministério da Saúde (BRASIL, 2000).

Em nenhuma das amostras analisadas foi detectada a presença de Salmonella spp. em $25 \mathrm{~g}$, coliformes totais, coliformes fecais, coliformes fecais termotolerantes (NMP/g) e de Staphylococcus coagulase positiva (UFC/g), atendendo a recomendação da RDC 12, de 2 de janeiro de 2001 (BRASIL, 2001). Demonstrou-se assim que o concentrado de melancia não apresentou microorganismos patogênicos e nem deteriorantes, sendo produzido de acordo com as Boas Práticas de Fabricação e que o tratamento térmico foi adequado para garantir qualidade higiênico-sanitária satisfatória ao produto. Segundo Franco e Landgraf (2003), o emprego de tratamentos térmicos reduz a carga microbiana dos alimentos devido aos efeitos deletérios que o calor exerce sobre grande parte dos micro-organismos. 


\subsection{ANÁLISE SENSORIAL}

Os atributos sensoriais avaliados diferiram estatisticamente $(p<0,05)$, exceto o aroma e a consistência. A qualidade global e a intenção de compra foram semelhantes entre as amostras do concentrado de melancia adicionado ou não de gelatina (Tabela 1).

\section{TABELA 1 - ESCORES MÉDIOS DOS TESTES DE ACEITAÇÃO SENSORIAL E INTENÇÃO DE COMPRA REALIZADOS COM DOIS TIPOS DE CONCENTRADO DE MELANCIA (Citrullus v. SCHRAD)}

\begin{tabular}{ccc}
\hline & \multicolumn{2}{c}{ AMOSTRAS } \\
\cline { 2 - 3 } ATRIBUTOS* $^{*}$ & $\begin{array}{c}\text { Concentrado de } \\
\text { melancia }\end{array}$ & $\begin{array}{c}\text { Concentrado de melancia } \\
\text { com gelatina }\end{array}$ \\
\hline Aparência & $7,70 \pm 1,15 \mathrm{a}$ & $5,63 \pm 1,65 \mathrm{~b}$ \\
Cor & $8,32 \pm 0,78 \mathrm{a}$ & $5,92 \pm 1,79 \mathrm{~b}$ \\
Aroma & $5,83 \pm 1,58 \mathrm{a}$ & $5,90 \pm 1,54 \mathrm{a}$ \\
Textura & $6,58 \pm 1,37 \mathrm{a}$ & $5,86 \pm 1,78 \mathrm{~b}$ \\
Consistência & $6,70 \pm 1,68 \mathrm{a}$ & $6,90 \pm 1,93 \mathrm{a}$ \\
Sabor & $6,22 \pm 1,99 \mathrm{a}$ & $5,57 \pm 1,87 \mathrm{~b}$ \\
Sabor residual & $5,72 \pm 1,81 \mathrm{a}$ & $4,85 \pm 1,86 \mathrm{~b}$ \\
Qualidade Global & $6,80 \pm 1,62 \mathrm{a}$ & $6,83 \pm 1,75 \mathrm{a}$ \\
Intenção de compra** & $4,27 \pm 1,06 \mathrm{a}$ & $3,14 \pm 1,21 \mathrm{~b}$ \\
\hline
\end{tabular}

*Escala com 9 pontos; **Escala com 5 pontos. Médias \pm desvio-padrão com letras diferentes na mesma linha diferiram entre si pelo teste de $t$ de Student $(p<0,05)$.

O aroma característico da melancia é produzido por aldeídos com nove carbonos e álcoois, os quais após tratamento térmico podem sofrer alterações em relação ao aroma da fruta in natura (THOMAZINI e FRANCO, 2000; XISTO et al., 2012). O aroma dos dois tipos de concentrado mostrou-se semelhante, entretanto alguns julgadores relataram aroma menos pronunciado de melancia nesses produtos, o que corrobora o estudo de Aguiló-Agayo et al. (2010) que observaram a diminuição do aroma e do sabor com o processamento e o armazenamento de suco de melancia.

O concentrado de melancia sem adição de gelatina apresentou boa aceitação, enquadrada entre "gostei ligeiramente" e "gostei muito", e maiores notas sensoriais para os atributos cor, sabor, sabor residual menos pronunciado e textura que o concentrado adicionado de gelatina.

O sabor da melancia é determinado pela interação entre ácidos orgânicos, açúcares, taninos e compostos de enxofre que podem ser alterados pelo tratamento térmico. A coloração vermelha da polpa da melancia decorre da presença de licopeno (LEÃO, PEIXOTO e VIEIRA, 2006; LIMA NETO et al., 2010) que pode ter influenciado positivamente a cor final do concentrado. Esse composto tem sua biodisponibilidade aumentada (ZHANG et al., 2011) pelo tratamento térmico em decorrência da isomerização da forma isomérica trans para cis, conforme relatado por Knockaert et al. (2012).

O concentrado de melancia mostrou aceitação sensorial semelhante a outros produtos elaborados com a polpa ou a entrecasca da melancia, como doce (SANT'ANA e OLIVEIRA, 2005), biscoitos (GUIMARÃES et al., 2012), bolos (GUIMARÃES, FREITAS e SILVA, 2010) e melancia minimamente processada e irradiada (MARTINS et al., 2008), apresentando notas sensoriais iguais ou acima de 7.

Tendo em vista as maiores notas sensoriais e de intenção de compra apresentadas pelo concentrado de melancia sem adição de gelatina, essa amostra foi selecionada para a realização das análises físico-químicas e do screening fitoquímico. 


\subsection{ANÁLISES FÍSICO-QUÍMICAS}

$\mathrm{Na}$ Tabela 2 observa-se que o concentrado de melancia apresentou composição centesimal e valor calórico diferente $(p<0,05)$ dos determinados na polpa e entrecasca da fruta in natura.

\section{TABELA 2 - ANÁLISES FÍSICO-QUÍMICAS DA POLPA E ENTRECASCA DA MELANCIA (Citrullus v. SCHRAD) E DO CONCENTRADO ELABORADO}

\begin{tabular}{ccc}
\hline DETERMINAÇõES & Polpa e entrecasca & Concentrado de melancia \\
\hline Umidade (\%) & $92,36 \pm 0,37 \mathrm{a}$ & $73,29 \pm 2,60 \mathrm{~b}$ \\
Extrato Mineral Fixo (\%) & $0,62 \pm 0,14 \mathrm{~b}$ & $1,03 \pm 0,10 \mathrm{a}$ \\
Proteínas (\%) & $0,79 \pm 0,11 \mathrm{a}$ & $0,55 \pm 0,01 \mathrm{~b}$ \\
Extrato Etéreo (\%) & $\mathrm{nd}^{*}$ & $1,08 \pm 0,10$ \\
Carboidratos (\%) & $6,22 \pm 0,31 \mathrm{~b}$ & $23,99 \pm 2,70 \mathrm{a}$ \\
Valor Energético (\%) & $28,05 \pm 1,24 \mathrm{~b}$ & $104,86 \pm 10,20 \mathrm{a}$ \\
pH & $5,44 \pm 0,03 \mathrm{~b}$ & $6,09 \pm 0,06 \mathrm{a}$ \\
Acidez (\%) & $0,20 \pm 0,4 \mathrm{a}$ & $0,14 \pm 0,3 \mathrm{~b}$ \\
Pectina (\%) & $0,22 \pm 0,1 \mathrm{~b}$ & $0,43 \pm 0,2 \mathrm{a}$ \\
\hline
\end{tabular}

Médias \pm desvio-padrão com letras diferentes na mesma linha diferiram entre si pelo teste de $t$ de Student $(p<0,05)$. ${ }^{*}$ nd = não detectado.

O concentrado, quando comparado com a fruta in natura apresentou menor percentual de umidade e proteína $(73,29 \%$ e $0,55 \%$, respectivamente). O tratamento térmico concentrou os carboidratos e as cinzas, ocorrendo elevação no percentual de lipídios (não detectado na fruta in natura). A polpa e a entrecasca da melancia apresentaram maiores teores de umidade e menores de carboidrato e de valor calórico em comparação à Tabela Brasileira de Composição de Alimentos (TACO, 2006). No entanto, evidenciaram maior teor proteico e menor teor lipídico quando comparados aos da fruta in natura de $0,5 \%$ de proteínas e $0,43 \%$ de lipídios (FAO, 2000). Vale ressaltar que esses valores podem variar de uma região para outra, tendo em vista que são dependentes do estádio de maturação, condições de adubação do local de plantio, fatores climáticos e condições genéticas da planta (MENDES, 2001).

O concentrado de melancia apresentou composição centesimal diferente da farinha da entrecasca de melancia analisada por Guimarães, Freitas e Silva (2010), pois o tratamento térmico concentrou os teores de cinzas e principalmente de carboidratos e proteínas, alcançando maior valor calórico. Wu et al. (2007) determinaram $24 \%$ de carboidratos em suco de melancia, resultado semelhante ao do concentrado (23,99\%).

$\mathrm{O}$ concentrado apresentou menor ácidez $(0,20 \%)$ e pH $(5,44 \%)$ maior que a fruta in natura, indicando que o tratamento térmico aplicado para sua elaboração pode ter degradado alguns ácidos orgânicos. $\mathrm{O}$ valor de pH obtido está em consonância com a pesquisa realizada com geleia de melancia (FERREIRA et al., 2010) e melancia minimamente processada (MIGUEL, DIAS e SPOTO, 2007), caracterizando produto pouco ácido ( $\mathrm{pH}>4,5)$. O concentrado de melancia também evidenciou baixa acidez, com acidez titulável em solução normal semelhante à acidez da geleia de melancia (FERREIRA et al., 2010). A natureza e concentração dos ácidos orgânicos são de grande interesse devido sua influência sobre as propriedades sensoriais e estabilidade de produtos à base de frutas (SCHERER et al., 2012) 
Constatou-se a ausência de pectina na polpa, já na entrecasca os valores variaram entre $0,21 \%$ e $0,23 \%$. O produto processado apresentou teor de pectina superior ao da entrecasca (elevação de 51,2 \%). Em estudo realizado com melancia minimamente processada por Miguel, Dias e Spoto (2007), as porcentagens de pectina solúvel e total sofreram influência da aplicação de cloreto de cálcio e do período de armazenamento. Os teores de pectina total foram estatisticamente mais elevados nos cubos de melancia tratados com cálcio e o armazenamento provocou ligeiro incremento nesse teor. A pectina constitui hidrocoloide importante para a indústria de alimentos, segundo Paiva, Lima e Paixão (2009), pois está associada à firmeza dos frutos, propriedades de formação de gel e aumento da viscosidade quando adicionada aos alimentos. A pectina deve estar presente na proporção mínima de 0,1\% para atender essas propriedades, percentual condizente com o verificado na entrecasca e no concentrado de melancia elaborado.

No teste de correlação entre os três açúcares presentes no concentrado constatou-se correlação negativa entre os açúcares redutores em glicose e os não redutores em sacarose e entre os açúcares redutores em glicose e a frutose (Tabela 3). No entanto, verificou-se correlação positiva entre a sacarose e a frutose.

\section{TABELA 3 - COEFICIENTES DE CORRELAÇÃO ENTRE OS AÇÚCARES PRESENTES NO CONCENTRADO DE MELANCIA E TEORES MÉDIOS DE AÇÚCARES REDUTORES E NÃO REDUTORES DO CONCENTRADO DE MELANCIA}

\begin{tabular}{cccc|c}
\hline Correlações & Glicose & Sacarose & Frutose & $\begin{array}{c}\text { Teor de açúcar no } \\
\text { concentrado de melancia } \\
(\mathbf{m g} / \mathbf{m L})\end{array}$ \\
\hline Glicose & 1,00 & $-0,22$ & $-0,03^{*}$ & $13,34 \pm 3,154$ \\
Sacarose & $-0,22$ & 1,00 & $+0,06$ & $23,69 \pm 1,315$ \\
Frutose & $-0,03^{*}$ & $+0,06$ & 1,00 & $10,34 \pm 4,427$ \\
\hline
\end{tabular}

* significativo ao nível de $5 \%(p<0,05)$.

As correlações verificadas são justificadas pelo grau de maturidade da fruta. As mais maduras apresentam maior teor de açúcares solúveis, principalmente da sacarose, proveniente tanto do acúmulo mediante a fotossíntese como pela hidrólise de carboidratos de reserva como o amido (CHITARRA e CHITARRA, 2005).

O concentrado de melancia apresentou menores teores de glicose, sacarose e frutose que o suco de melancia analisado por Quek, Chok e Swedlund (2007), que determinaram valores de $55,31 \mathrm{mg} / \mathrm{mL}$ para glicose, $11,70 \mathrm{mg} / \mathrm{mL}$ para sacarose e $68,53 \mathrm{mg} / \mathrm{mL}$ para frutose. $O$ tratamento térmico aplicado ao concentrado de melancia pode ter ocasionado perdas de açúcares solúveis durante o processamento (MESQUITA et al., 2003; QUEK, CHOK e SWEDLUND, 2007). Além disso, a concentração de açúcares dos frutos depende das condições ambientais, pois excesso de água no estádio final de maturação pode resultar em frutos pouco doces, devido maior diluição dos açúcares (CASTELLANE e CORTEZ, 1995).

\subsection{SCREENING FITOQUÍMICO}

Os testes fitoquímicos mostraram o grau de intensidade da presença dos compostos fitoquímicos no concentrado de melancia. Conforme explicitado na Tabela 4 pode-se inferir que o princípio ativo do concentrado está baseado em esteroides e flavonoides, caracterizando o concentrado e a melancia como fontes desses fitonutrientes (PERKINS-VEAZIE e COLLINS, 2004). 
TABELA 4 - SCREENING FITOQUÍMICO DA MELANCIA IN NATURA E DO CONCENTRADO DE MELANCIA ELABORADO

\begin{tabular}{|c|c|c|c|c|c|}
\hline \multicolumn{2}{|c|}{ COMPOSTOS FITOQUÍMICOS } & \multicolumn{4}{|c|}{ AMOSTRAS } \\
\hline Grupo Químico & $\begin{array}{c}\text { Métodos/ } \\
\text { Concentrações }\end{array}$ & Semente & Polpa & Entrecasca & Concentrado \\
\hline \multirow{3}{*}{ Esteroides } & 0,12 & - & + & - & - \\
\hline & 0,25 & - & + & + & + \\
\hline & 0,50 & - & ++ & + & + \\
\hline \multirow{4}{*}{ Taninos } & Gelatina $\quad 1,0$ & - & - & - & - \\
\hline & 2,0 & - & + & - & + \\
\hline & $\mathrm{FC}_{3} 2 \% \quad 1,0$ & - & + & - & + \\
\hline & 2,0 & - & + & + & + \\
\hline \multirow{2}{*}{ Saponinas } & Hemolítico & - & - & + & - \\
\hline & Espuma & + & - & - & - \\
\hline \multirow{2}{*}{ Flavonoides } & Fita Magnésio & ++ & ++ & + & - \\
\hline & Fluorescência & ++ & ++ & + & + \\
\hline
\end{tabular}

Legenda: (-) Reação Negativa; (+) Reação fracamente positiva; (++) Reação positiva.

A polpa da melancia demonstrou maior concentração de compostos fitoquímicos quando comparada às outras partes dessa fruta e ao concentrado elaborado que também apresentou esteroides, taninos e flavonoides em menor intensidade. Segundo Pereira, Miguel e Carvalho (2010), Tlili et al. (2011) e Rawson et al. (2011), o genótipo, o estádio de maturação, o processamento e a estocagem de melancias e de outras frutas influenciam seu teor de compostos bioativos.

Sob o ponto de vista funcional, os principais esteroides encontrados em alimentos vegetais são os fitoesterois que atuam na diminuição da absorção do colesterol no intestino delgado mediante mecanismo de competição, com consequente aumento da excreção fecal e efeitos na prevenção e tratamento de doenças cardiovasculares (TASAN et al., 2006; BRUFAU, CANELA e RAFECAS, 2008).

A polpa da melancia mostrou reação positiva para a presença de tanino, mas o produto elaborado apresentou menor intensidade desse composto. Tal fato pode ser benéfico se o tanino for considerado fator antinutricional ao formar complexos insolúveis com minerais, proteínas e amidos, tornando-os biologicamente indisponíveis para seres humanos em condições fisiológicas normais (MARTINEZ-VALVERDE, PERIAGO e ROS, 2000; MARTINEZ-DOMINGUEZ, IBAÑEZ e RINCÓN, 2002). No entanto, os taninos apresentam atividade antioxidante, sendo considerados compostos bioativos (BRAVO, GOYA e LECUMBERRI, 2007; SILVA et al., 2008).

A ausência de saponinas na composição do concentrado aumenta seu potencial de utilização e consumo, tendo em vista que esses compostos são considerados fatores antinutricionais. Quando consumidos reduzem o valor nutritivo dos alimentos, interferindo na digestibilidade, absorção ou utilização de nutrientes e em altas concentrações podem acarretar efeitos danosos à saúde (SANTOS, 2006), como a redução da função hepática em animais (MELO et al., 2008). Diversos estudos foram conduzidos objetivando sua quantificação (OJIAKO e IGWE, 2007; SEMBRATOWICZ et al., 2008) e a redução da concentração desses compostos (MUKHOPADHYAY, SARKAR, BANDYOPADHYAY, 2007), demonstrando sua importância para a ciência dos alimentos. 
Observou-se perda de flavonoides após o tratamento térmico e produção do concentrado de melancia, tendo em vista que esses compostos podem ter seu teor reduzido em decorrência da trituração, prensagem, aplicação de calor ou frio e durante o armazenamento (PEREIRA, MIGUEL e CARVALHO, 2010; OMS-OLIU et al., 2012). Estudos demonstraram a ação anti-inflamatória dos flavonoides devido à sua capacidade para eliminar oxigênio e espécies reativas, além de elevada atividade antioxidante in vitro e in vivo (IZZI et al., 2012), evidenciando a necessidade do controle efetivo das etapas de processamento dos alimentos, em particular das frutas, para minimizar as perdas desses e de outros fitonutrientes.

\section{CONCLUSÃO}

A produção do concentrado de melancia mostrou-se viável devido à ampla disponibilidade da fruta. Apesar do baixo rendimento, a elaboração desse produto justifica-se pelo aproveitamento de resíduos da pós-colheita da melancia, alta perecibilidade da fruta in natura e maior vida-deprateleira do concentrado, bem como pelas suas características sensoriais, nutricionais e a presença de compostos fitoquímicos com propriedades antioxidantes. Em decorrência de o concentrado de melancia apresentar nutrientes com características funcionais, seu uso no contexto terapêutico pode ser viabilizado e constituir opção dentre os alimentos funcionais necessários à dieta saudável.

\section{ABSTRACT \\ WATERMELON CONCENTRATE (Citrullus vulgaris Schrad): SENSORY ACCEPTANCE, MICROBIOLOGICAL AND PHYSICOCHEMICAL PARAMETERS AND DETERMINATION OF PHYTONUTRIENTS}

The objective of this study was to produce watermelon concentrate and determine its sensory acceptability, microbiological and physicochemical parameters and content of phytonutrients. Pulp and bast were crushed and homogenized before being submitted to heat treatment to obtain the concentrate. A portion of this concentrate was added to red unflavored gelatin. The samples were cooled and stored at refrigeration temperature. Microbiological analysis was carried out and subsequently, sensory analysis of watermelon concentrated and watermelon concentrate plus gelatin was performed. From these analyses, pure concentrate was selected for the determination of physicochemical parameters and phytochemical screening of the fresh fruit. Both concentrates showed microbiological quality in accordance with Brazilian current legislation. Concentrate with no added gelatin was selected for the other analyses due to its good acceptance and highest scores in the sensory attributes of color, flavor, aftertaste and texture. When compared to fresh fruit, concentrates showed lower moisture, protein, pectin and acidity percentages. However, the heat treatment concentrated carbohydrate and ash, $23.99 \%$ and $1.03 \%$, respectively, increasing the calorific value from 28.05 to $104.86 \mathrm{kcal}$. The active ingredient of the watermelon concentrate is based on steroids and flavonoids. The production of watermelon concentrate proved to be feasible due to the wide availability of fruit and to its sensory and nutritional characteristics and to the presence of phytochemicals with antioxidant properties. This product not only shows longer shelf life when compared to the fresh fruit, but is also an important option among functional foods required for a healthy diet.

KEY-WORDS: WATERMELON; SENSORY ANALYSIS; PHYTOCHEMICALS; WATERMELON CONCENTRATE.

\section{REFERÊNCIAS}

1 AGUILÓ-AGUAYO, I.; MONTERO-CALDERÓN, M.; SOLIVA-FORTUNY, R.; MARTíN-BELLOSO, O. Changes on flavor compounds throughout cold storage of watermelon juice processed by high-intensity pulsed electric fields or heat. Journal of Food Engineering, v.100, n.1, p.43-49, 2010.

2 AHN, J.; CHOI, W.; KIM, S.; HA, T. Anti-diabetic effect of watermelon (Citrullus vulgaris Schrad) on streptozotocin-induced diabetic mice. Food Science and Biotechnology, v.20, n.1, p.251-254, 2011.

3 ARAÚJO, I.M.L. Avaliação do efeito hipoglicemiante da Citrullus vulgaris Schrad (Melancia) em indivíduos diabéticos tipo 2 e normais. 1999. 134 f. Dissertação (Mestrado em Ciências da Nutrição), Universidade Federal da Paraíba (UFPB), João Pessoa, 1999. 
4 BARBOSA FILHO, J.M. Triagem fitoquímica de plantas medicinais do estado da Paraíba. Boletim da Sociedade Broteriana, v.2, n.57, p.1-9, 1997.

5 BRASIL. Ministério da Saúde. Agência Nacional de Vigilância Sanitária. RDC 63, de 6 de julho de 2000. Aprova o regulamento técnico para fixar os requisitos mínimos exigidos para terapia de nutrição enteral. Diário Oficial [da] República Federativa do Brasil, Brasília, 2000. Seção 1, p. 2284.

6 BRASIL. Ministério da Saúde. Agência Nacional de Vigilância Sanitária. RDC 12 de 2 de janeiro de 2001. Aprova o regulamento técnico sobre padrões microbiológicos para alimentos. Diário Oficial [da] República Federativa do Brasil, Brasília, 10 jan. 2001, Seção 1, p. 45-53.

7 BRASIL. Ministério da Saúde. Resolução RDC 360 de 23 de dezembro de 2003. Aprova o regulamento técnico para rotulagem nutricional obrigatória de alimentos e bebidas embalados. Diário Oficial [da] República Federativa do Brasil, Brasília, DF, 26 dez. 2003. Seção 1, p.33-34.

8 BRASIL. Ministério da Saúde. Agência de Vigilância Sanitária. Métodos físico-químicos para análise de alimentos. Brasília (DF): Instituto Adolfo Lutz, 2005.

9 BRAVO, L.; GOYA, L.; LECUMBERRI, E. LC/MS characterization of phenolic constituents of mate (Ilex paraguariensis, St. Hil.) and its antioxidant activity compared to commonly consumed beverages. Food Research International, v.40, n.3, p.393-405, Apr. 2007.

10 BRUFAU, G.; CANELA, M.A.; RAFECAS, M. Phytosterols: physiologic and metabolic aspects related to cholesterollowering properties. Nutrition Research, v.28, n.4, p.217-225, 2008.

11 CARVALHO, R.N. Cultivo de melancia para a agricultura familiar: serviço de produção de informação. Brasília, DF: EMBRAPA, 1999. $127 \mathrm{p}$

12 CASTELLANE, P.D.; CORTEZ, G.E. A cultura da melancia. Jaboticabal: FUNEP/FCAV-UNESP, 1995.64 p.

13 CHITARRA, M.I.F.; CHITARRA, A.B. Pós-colheita de frutos e hortaliças: fisiologia e manuseio. 2. ed. Lavras: UFLA, 2005. $785 \mathrm{p}$.

14 DIAS, R.C.S.; SILVA, C.M.J.; QUEIROZ, M.A.; COSTA, N.D.; SOUZA, F.F.; SANTOS, M.H.; PAIVA, L.B.; BARBOSA, G.S.; MEDEIROS, K.N. Desempenho agronômico de linhas de melancia com resistência ao oídio. Horticultura Brasileira, v.24, p.1416-1418, 2006

15 Food and Agriculture Organization (FAO). Sandia: composición nutricional. 2006. Disponível em: <http://www.fao.org/ inpho_archive/content/documents/vlibrary/AE620s/Pfrescos/SANDIA.HTM > Acesso em: 20 de maio 2012.

16 FERREIRA, R.M.A.; AROUCHA, E.M.M.; SOUSA, A.E.D.; MELO, D.R.M.; PONTES FILHO, F.S.T. Processamento e conservação de geléia mista de melancia e tamarindo. Revista Verde, v.5, n.3, p.59-62, 2010.

17 FIGUEROA, A.; SANCHEZ-GONZALEZ, M.A.; WONG, A.; ARJMANDI, B.H. Watermelon extract supplementation reduces ankle blood pressure and carotid augmentation index in obese adults with prehypertension or hypertension. American Journal of Hypertension, v.25, n.6, p.640-643, 2012.

18 FRANCO, B.D.G.M.; LANDGRAF, M.L.S. Microbiologia dos alimentos. São Paulo: Atheneu, 2003.182 p.

19 GUIMARÃES, R.R.; FREITAS, M.C.J.; SILVA, V.L.M. Bolos simples elaborados com farinha da entrecasca de melancia (Citrullus vulgaris, Sobral): avaliação química, física e sensorial. Ciência e Tecnologia de Alimentos, Campinas, v. 30, n.2, p.354-363, 2010.

20 GUIMARÃES, R.R.; GAMA, C.R.; FREITAS, M.C.J.; SILVA, V.L.M. Biscoitos sequilhos de cebola elaborados com farinha da entrecasca de melancia (Citrullus vulgaris). Sociedade Brasileira de Processamento de Frutas e Hortaliças. Disponível em: http://sbpfh.org.br Acesso em: 14 de maio 2012.

21 Instituto Brasileiro de Geografia e Estatística (IBGE). Produção agrícola municipal: lavoura temporária. Disponível em: http://seriesestatisticas.ibge.gov.br/series.aspx?vcodigo=PA3\&sv=83\&t=lavouratemporaria-quantidade-produzida. Acesso em: 10 maio 2012.

22 IZZI, V.; MASUELLI, L.; TRESOLDI, I.; SACCHETTI, P.; MODESTI, A.; GALVANO, F.; BEI, R. The effects of dietary flavonoids on the regulation of redox inflammatory networks. Frontiers in Bioscience, v.17, p.2396-2418, 2012.

23 JANDEL SCIENTIFIC. Sigma stat. Version 3.5. San Jose, CA, 2007.

24 KNOCKAERT, G.; PULISSERY, S.K.; COLLE, I.; BUGGENHOUT, S.V.; HENDRICK, X.M.; VAN LOEY, A. Lycopene degradation, isomerization and in vitro bioaccessibility in high pressure homogenized tomato puree containing oil: effect of additional thermal and high pressure processing. Food Chemistry,v.135, p.1290-1297, 2012.

25 LEÃO, D.S.; PEIXOTO, J.R.; VIEIRA, J.V. Teor de licopeno e de sólidos solúveis totais em oito cultivares de melancia. Biosciences Journal, v.22, n.3, p.7-15, 2006. 
26 LIMA NETO, I.S.; GUIMARÃES, I.P.; BATISTA, P.F.; AROUCHA, E.M.M.; QUEIROZ, M.A. Qualidade de frutos de diferentes variedades de melancia provenientes de Mossoró-RN. Revista Caatinga, v. 23, n. 4, p. 14-20, 2010.

27 LUENGO, R.F.A.; PARMAGNANI, R.M.; PARENTE, M.R.; LIMA, M.F.B.F. Tabela de composição nutricional das hortaliças. Brasília: EMBRAPA Hortaliças. 2000. 4 p.

28 MARRERO, A.; KADER, A.A. Optimal temperature and modified atmosphere for keeping quality of fresh-cut pineapples. Postharvest Biology and Technology, v.39, p.161-168, 2006.

29 MARTINS, C.G; ARAGON-ALEGRO, L.C.; BEHRENS, J.H.; SOUZA, K.L.O.; VIZEU, D.M.; HUTZLER, B.W.; DESTRO, M.T.; LANDGRAF, M. Acceptability of minimally processed and irradiated pineapple and watermelon among Brazilian consumers. Radiation Physics and Chemistry, v.77, p.825-829, 2008.

30 MARTINEZ-VALVERDE, I.; PERIAGO, M.J; ROS, G. Significado nutricional de los compuestos fenólicos de la dieta. Archivos Latinoamericanos de Nutrición, v.50, n.1, p. 5-18, 2000.

31 MARTINEZ-DOMINGUEZ, B.; IBAÑEZ, M.; RINCÓN, F. Acido fítico: aspectos nutricionales e implicaciones analíticas. Archivos Latinoamericanos de Nutrición, v.52, n.3, p.219-231, 2002.

32 MEILGAARD M.; CIVILLE G.V.; CARR B.T. Consumer test and in-house panel acceptance tests. In: SENSORY evaluation techniques. Florida: CRC Press, 1988.

33 MELO, D.S.; CORREAA, A.D.; MARCOS, F.C.A.; SOUSA, R.V.; ABREU, C.M.P.; SANTOS, C.D. Efeitos da farinha de folhas de mandioca sobre a atividade das enzimas AST, ALT, FA e lipídios hepáticos de ratos Wistar. Ciência e Tecnologia de Alimentos, v.28, p.32-37, 2008.

34 MENDES, M.H.M. Tabela de composição de alimentos: amiláceos, cereais e derivados, frutas, hortaliças, leguminosas, nozes e oleaginosa. Niterói: EDUFF, 2001. 41 p.

35 MESQUITA, P.C.; MAIA, G.A.; SOUZAFILHO, M.S.M.; NASSU, R.T. Estabilidade microbiológica, físico-química e sensorial de pedúnculos de caju (Anacardium occidentale L.) processados por métodos combinados. Ciência e Tecnologia de Alimentos, v.23, n.3, p. 366-369, 2003.

36 MIGUEL, A.C.A.; DIAS, J.R.P.S.; SPOTO, M.H.F. Efeito do cloreto de cálcio na qualidade de melancias minimamente processadas. Horticultura Brasileira, v. 25, p.442-446, 2007.

37 MUKHOPADHYAY, N.; SARKAR, S.; BANDYOPADHYAY, S. Effect of extrusion cooking on anti-nutritional factor tannin in linseed (Linum usitatissimum) meal. International Journal of Food Sciences and Nutrition, v.58, n.8, p.588-594, 2007.

38 OJIAKO, O.A.; IGWE, C.U. Nutritional and anti-nutritional compositions of Cleome rutidosperma, Lagenaria siceraria, and Cucurbita maxima seeds from Nigeria. Journal of Medicinal Food, v.10, n.4, p.735-738, 2007

39 OMS-OLIU, G.; ODRIOZOLA-SERRANO, I.; SOLIVA-FORTUNY, R.; MARTÍN-BELLOSO, O. Stability of health-related compounds in plant foods through the application of non thermal. Trends in Food Science and Technology, v.23, p.111123, 2012.

40 PAIVA, E.P.; LIMA, M.S.; PAIXÃO, J.A. Pectina: propriedades químicas e importância sobre a estrutura da parede celular de frutos durante o processo de maturação. Revista Iberoamericana de Polímeros, v.10, n.4, p.196-211, 2009.

41 PEREIRA, A.S.; MIGUEL, D.P.; CARVALHO, E.E.N. Caracterização de farinha da entrecasca de melancia (Citrullus lanatus) produzida na região sul do Tocantins. Cadernos de Pós-Graduação da FAZU, v.1, p.1-5, 2010.

42 PERKINS-VEAZIE, P.; COLLINS, J.K. Flesh quality and lycopene stability of fresh-cut watermelon. Postharvest Biology and Technology, v.31, p.159-166, 2004.

43 PINTO, S. A. A. Processamento mínimo de melão tipo Orange Flesh e de melancia 'Crimson Sweet'. 2002.120 f. Dissertação (Mestrado em Fitotecnia), Faculdade de Ciências Agrárias e Veterinárias, Universidade Estadual Paulista "Júlio Mesquita Filho", Jaboticabal, 2002.

44 QUEK, S.Y.; CHOK, N.K.; SWEDLUND, P. The physicochemical properties of spray-dried watermelon powders. Chemical Engineering and Processing, v.46, p.386-392, 2007.

45 RANGANNA, S. Handbook of analysis and quality control for fruit and vegetable products. New Delhi: McGraw-Hill, 1979.

46 RAWSON, A.; TIWARI, B.K.; PATRAS, A.; BRUNTON, N.; BRENNAN, C.; CULLEN, P.J.; O’DONNEL, C. Effect of thermosonication on bioactive compounds in watermelon juice. Food Research International, v.44, p.1168-1173, 2011.

47 SANT'ANA, A.F.; OLIVEIRA, L.F. Aproveitamento da casca de melancia (Curcubita citrullus, Schrad) na produção artesanal de doces alternativos. Alimentos e Nutrição, Araraquara, v.16, n.4, p. 363-368, out./ dez. 2005.

48 SANTOS, M.A.T. Efeito do cozimento sobre alguns fatores antinutricionais em folhas de brócolis, couve-flor e couve. 
Ciência e Agrotecnologia, v.30, n.2, p. 294-301, 2006.

49 SCHERER, R.; RYBKA, A.C.P.; BALLUS, C.A.; MEINHART, A.D.; TEIXEIRA FILHO, J.; GODOY, H.T. Validation of a HPLC method for simultaneous determination of main organic acids in fruits and juices. Food Chemistry, v.135, n.1, p.150-154, nov. 2012.

50 SEMBRATOWICZ, I.; OGNIK, K.; RUSINEK, E.; TRUCHLIŃSKI, J. Contents of tannins and oxalic acid in the selected forest fruits depending on the harvest site. Rocz Panstw Zakl Hig, v.59, n.1, p.41-46, 2008.

51 SILVA, E.L.; NEIVA, T.J.C.; SHIRAI, M.; TERAO, J.; ABDALLA, D.S.P. Acute ingestion of yerba mate infusion (Ilex paraguariensis) inhibits plasma and lipoprotein oxidation. Food Research International, v.41, p.973-979, 2008.

52 TABELA brasileira de composição de alimentos (TACO). 2. ed. Campinas: NEPA - UNICAMP, 2006. v.2. Disponível em: http://www.unicamp.br/nepa/taco Acesso em: 01 julho 2012.

53 TAŞAN, M.; BILGIN, B.; GEÇGEL, Ü.; DEMIRCI, A.Ş. Phytosterols as functional food ingredients. Journal of Tekirdag Agricultural Faculty, v.3, n.2, p.153-9, 2006.

54 THOMAZINI, M.; FRANCO, M.R.B. Metodologia para análise dos constituintes voláteis do sabor. Boletim da Sociedade Brasileira de Ciência e Tecnologia de Alimentos, v.34, n.1, p. 52-59, 2000.

55 TLILI, I.; HDIDER, C.; LENUCCI, M.S.; ILAHY, R.; JEBARI, H.; DALESSANDRO, G. Bioactive compounds and antioxidant activities during fruit ripening of watermelon cultivars. Journal of Food Composition and Analysis, v. 24, p.923-928, 2011.

56 VANDERZANT, C.; SPLITTSTOESSER, D.F. Compendium of methods for the microbiological examination of foods. $3^{\text {rd }}$ ed. Washington: American Public Health Association (APHA), 1992. 1219 p.

57 XISTO, A.L.R.P.; VLIAS BOAS, E.V.B.; NUNES, E.E.; VILAS BOAS, B.M.; GUERREIRO, M.C. Perfil volátil e alterações físicas, químicas e bioquímicas na melancia minimamente processada durante o armazenamento. Ciência e Tecnologia de Alimentos, v.32, n.1, p.173-178, 2012.

58 ZHANG, C.; TRIERWEILER, B.; LI, W.; BUTZ, P.; XU, Y.; RÜFER, C.E.; MA, Y.; ZHAO, X. Comparison of thermal, ultraviolet-c, and high pressure treatments on quality parameters of watermelon juice. Food Chemistry, v.126, p.254260, 2011.

59 WU, G.; COLLINS, J.K.; PERKINS-VEAZIE, P.; SIDDIQ, M.; DOLAN, K.D.; KELLY, K.A.; HEAPS, C.L.; MEININGER, C.J. Dietary supplementation with watermelon pomace juice enhances arginine availability and ameliorates the metabolic syndrome in zucker diabetic fatty rats. The Journal of Nutrition, v.137, p.2680-2685, 2007.

\section{AGRADECIMENTOS}

Os autores agradecem à Coordenação de Aperfeiçoamento de Pessoal de Nível Superior (CAPES) pela bolsa de mestrado e ao Conselho Nacional de Desenvolvimento Científico e Tecnológico (CNPq) pelo financiamento da pesquisa. 